\title{
Challenges in the Management of Ectopic Parathyroid Pathologies: A Case Series of Five Patients
}

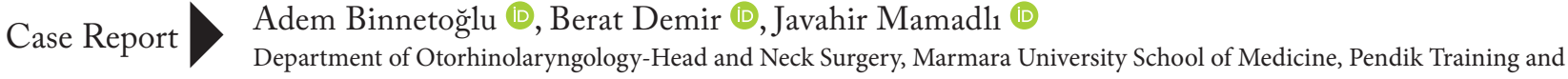 \\ Research Hospital, İstanbul, Turkey
}

\begin{abstract}
In primary hyperparathyroidism (PHPT) the diagnosis and treatment of ectopic parathyroid adenomas (EPTA) is a challenging process for head and neck surgeons. We present five patients with EPTA in different locations, along with an in-depth discussion of imaging modalities. We used sestamibi scintigraphy (MIBI) and ultrasound as first line imaging tools in asymptomatic hypercalcemia and PHPT. Single photon emission computed tomography (SPECT) was combined with MIBI or computed tomography (CT) if a parathyroid pathology was not localized initially. Four-dimensional parathyroid CT (4D-CT) was the last imaging modality preferred to localize the ectopic parathyroid gland. We performed focused unilateral
\end{abstract}

ORCID iDs of the authors:

A.B. 0000-0002-2797-0248;

B.D. 0000-0002-8574-4927;

J.M. 0000-0002-4907-3468

Cite this article as: Binnetoğlu A, Demir B, Mamadlı I. Challenges in the Management of Ectopic Parathyroid Pathologies: A Case Series of Five Patients. Turk Arch Otorhinolaryngol 2020; 58(2): 133-6.

\section{Corresponding Author:}

Berat Demir, drberatdemir80@hotmail.com

Received Date: 18.09 .2019

Accepted Date: 06.03 .2020

Content of this journal is licensed under a Creative Commons Attribution 4.0 International License. Available online at www.turkarchotolaryngol.net

\section{Introduction}

The use of routine serum calcium measurements in clinic practice has increased the number of diagnoses of asymptomatic hyperparathyroidism. A parathyroid adenoma (PTA) which secretes excess parathyroid hormone (PTH) increases the serum calcium (hypercalcemia) level and is the most common cause of primary hyperparathyroidism (PHPT) (1). Preoperatively, most adenomas can be localized with imaging methods like ultrasonography (USG) and technetium-99m-sestamibi scan or sestamibi scintigraphy (MIBI).

Ectopic parathyroid adenomas (EPTA) constitute approximately $22 \%$ of the cases with PHPT (2). Despite the advancements in imaging technologies and surgical techniques, the management of EPTA continues to pose challenges to both radiologists and surgeons. Developmental abnormalities of parathyroid gland migration results in ectopic neck exploration (FUNE) with intraoperative frozen section analysis as a routine procedure for imaging-detected lesions. Bilateral neck exploration was performed for re-exploration cases and imaging-negative cases. Histopathology confirmed EPTA and postoperative serum calcium normalized in all cases. 4D-CT is promising for ectopic parathyroid gland localization if all other imaging modalities fail. Despite the advancements in imaging, surgical experience continues to play the central role in the management of ectopic parathyroid pathologies.

Keywords: Hyperparathyroidism, parathyroid gland, ectopic parathyroid gland, nuclear radiology, four-dimensional computed tomography, surgery localization of parathyroid tissue in the superior mediastinum, thymus, carotid sheath, behind the esophagus or within the thyroid parenchyma itself (3). We present five EPTA cases together with their diagnostic and surgical challenges.

\section{Case Presentation}

\section{Case 1: Intrathymic PTA}

A 37-year-old man with nephrolithiasis was evaluated for PHPT. Blood analysis showed serum calcium of $10.7 \mathrm{mg} / \mathrm{dL}$ and PTH of $342.8 \mathrm{pg} /$ $\mathrm{mL}$. USG showed negative for parathyroid or thyroid abnormalities. MIBI showed tracer uptake in a thymic locus with early wash-out suggestive of EPT (Figure 1). A 1x1 cm mass was observed during operation in the thymus and was detected intraoperatively by gamma probe. The mass was excised together with the thymus. Frozen sections were consistent with parathyroid tissue. There was 
no postoperative hypocalcemia, and serum calcium dropped to $8.6 \mathrm{mg} / \mathrm{dL}$.

\section{Case 2: Paraesophageal PTA}

A 68-year-old woman with fatigue and memory loss was evaluated for PHPT. Her calcium in serum was $10.8 \mathrm{mg} / \mathrm{dL}$ and PTH was $156.3 \mathrm{pg} / \mathrm{mL}$. USG showed negative for parathyroid abnormalities. Preoperative localization with MIBI showed a retroesophageal focus. Minimally invasive parathyroidectomy was done. According to histopathologic examination, the tissue of the hypercellular parathyroid was consistent with an adenoma. The patient's symptoms resolved after surgery.

\section{Case 3: Intravagal PTA}

A 57-year-old woman who had three unsuccessful surgeries for hyperparathyroidism was investigated for ectopic PTA. Laboratuary results showed corrected serum calcium of $10.7 \mathrm{mg} / \mathrm{dL}$ and PTH of $1303 \mathrm{pg} / \mathrm{mL}$. USG showed negative for parathyroid abnormalities. Preoperative MIBI suggested right superior parathyroid pathology. Right superior parathyroid biopsy performed during the first surgery showed normal tissue. Postoperative MIBI suggested right inferior PTA. During the second surgery, exploration of right neck with right thyroid lobectomy showed negative for PTA. Biopsy was taken from the left inferoposterior thyroid mass. As specimen histology showed normal lymph node and the thyroid tissue. A third surgery for suspected left superior PTA showed mild to diffuse hyperplasia on histopathologic examination.

To detect EPTA, we requested magnetic resonance imaging (MRI) of the mediastinum and the neck. A $2 \times 1 \mathrm{~cm}$ mass was seen on the left neck at level 2 and confirmed as a lymph node on computed tomography (CT). According to MIBI, there was a suspicious accumulation of tracer at the left upper jugular level posterior to the submandibular gland (as shown in Figure 2). USG of the neck was unremarkable. A fourth surgical exploration was performed based on the above-mentioned findings. The left thyroid lobe was mobilized and explored, the upper mediastinum and the left paratracheal-paraesophageal groove were cleared, and the left recurrent laryngeal nerve was identified and preserved. A fusiform swelling $(2 \times 1 \mathrm{~cm})$ in the carotid sheath was identified in the vagus nerve at the carotid bifurcation level. The perineurium was opened and a tan-colored soft nodule was deprived of the nucleus from the vagus nerve. There were no complications during or after the operation such as hoarseness, bradycardia, or dysphagia. Histopathologic examination con-

\section{Main Points}

- Ectopic parathyroid tissue could be located in the superior mediastinum, thymus, carotid sheath, behind the esophagus or within the thyroid parenchyma.

- Technetium-99m-sestamibi scan or sestamibi scintigraphy (MIBI) and USG could be insufficient to detect ectopic parathyroid pathologies.

- 4D-CT scanning has advantages compared with the other modalities in patients with ectopic parathyroid pathologies. firmed EPTA. Postoperatively, the patient's serum calcium and PTH were $8.5 \mathrm{mg} / \mathrm{dL}$ and $7 \mathrm{pg} / \mathrm{mL}$, respectively.

\section{Case 4: PTA Posterior to Left Subclavian Artery}

A 51-year-old woman suffering from asymptomatic hypercalcemia was referred to our clinic. Her left thyroid lobe had been removed three years ago. Current serum calcium was $12.9 \mathrm{mg} / \mathrm{dL}$ and PTH was $63.9 \mathrm{pg} / \mathrm{mL}$. USG and MIBI were negative for parathyroid abnormalities. Physical examination of the neck was normal. Four-dimensional parathyroid CT (4D-CT) revealed a $15 \times 7 \mathrm{~mm}$ PTA which was near to the cranial cephalic artery in the left thyroid lobe inferior (Figure 3). A $1.5 \times 1 \mathrm{~cm}$ mass was excised with the surrounding rudimentary thymus tissue. Histopathologic examination confirmed EPTA. Postoperative serum calcium was $8.5 \mathrm{mg} / \mathrm{dL}$ and PTH decreased to $7.25 \mathrm{pg} / \mathrm{mL}$.

\section{Case 5: Intrathyroidal PTA}

A 56-year-old female with prior history of left lobo-isthmectomy presented with asymptomatic hypercalcemia. USG showed negative for parathyroid abnormalities. MIBI showed intense involvement (late phase) in right lower thyroid consistent with PTA. Serum calcium, however, remained high $(11.6 \mathrm{mg} / \mathrm{dL})$ after right inferior parathyroidectomy, and histopathologic examination was reported as normal parathyroid tissue. Repeat MIBI during the third postoperative month was negative; hence, the patient underwent a bilateral neck exploration (BNE) with complementary thyroidectomy. No abnormal mass was detected intraoperatively. After surgery her calcium level dropped (8.9 $\mathrm{mg} / \mathrm{dL}$ ) and histopathologic examination revealed the presence of an intrathyroidal PTA.

\section{Discussion}

The fourth pharyngeal pouch is the origin of the superior parathyroid glands that migrate down to its final position in the upper thyroid. The inferior pair arises from the third pouch which is along with thymus (4). During migration, the thymus drags the inferior pair down to the lower poles of the thyroid gland or the thyro-thymic ligament. Based on the migration path, the inferior pair can be seen anywhere from the base of tongue to the heart. Because of its longer migration path, the inferior pair shows more variability in its ectopic location than the superior pair. Common ectopic foci are posterior mediastinum, tracheoesophageal groove, anterior mediastinum, carotid sheath, retropharyngeal and retroesophageal positions, thyroid parenchyma, thymus, or even pericardium (4).

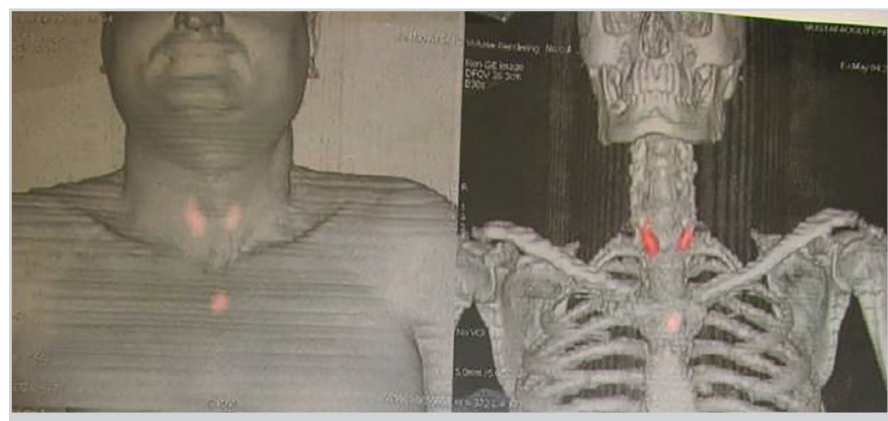

Figure 1. MIBI showed tracer uptake in a thymic locus with early wash-out suggestive of EPT 


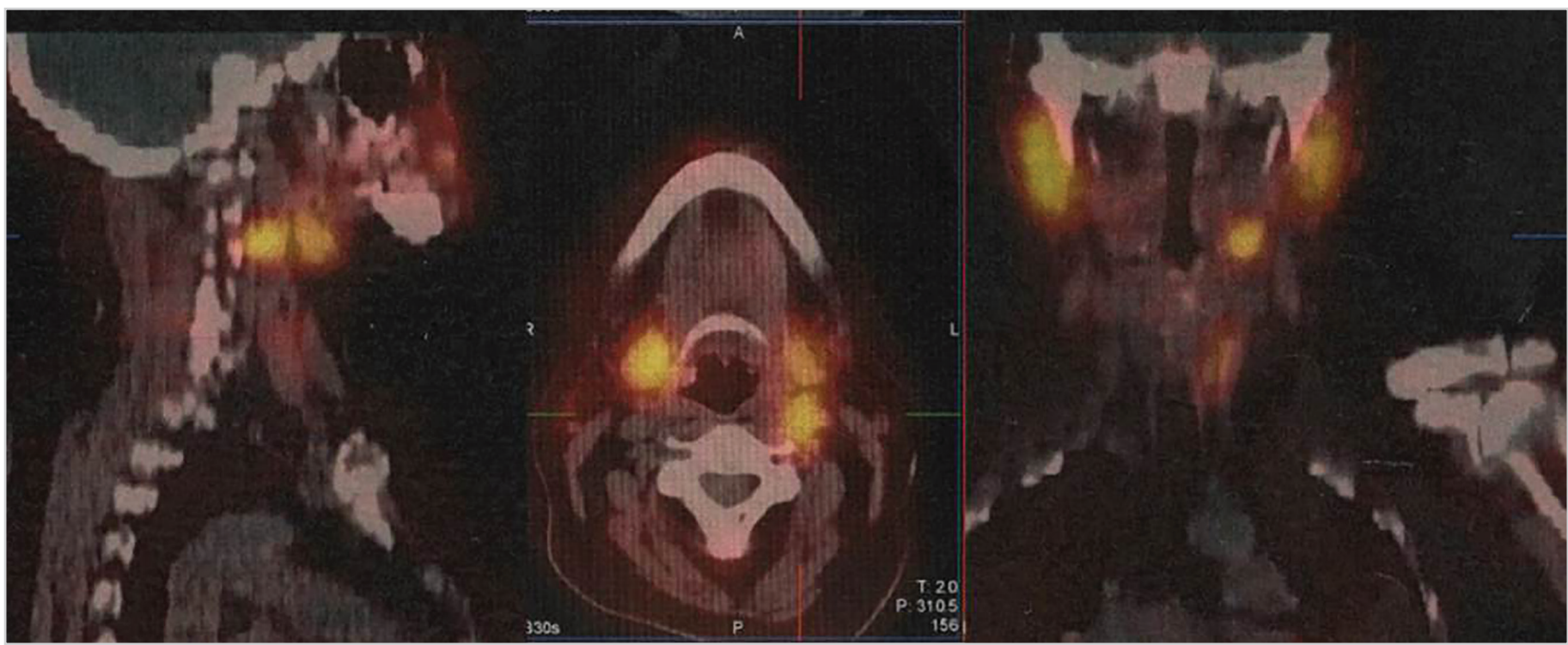

Figure 2. MIBI showed a suspicious tracer accumulation at left upper jugular level, posterior to submandibular gland

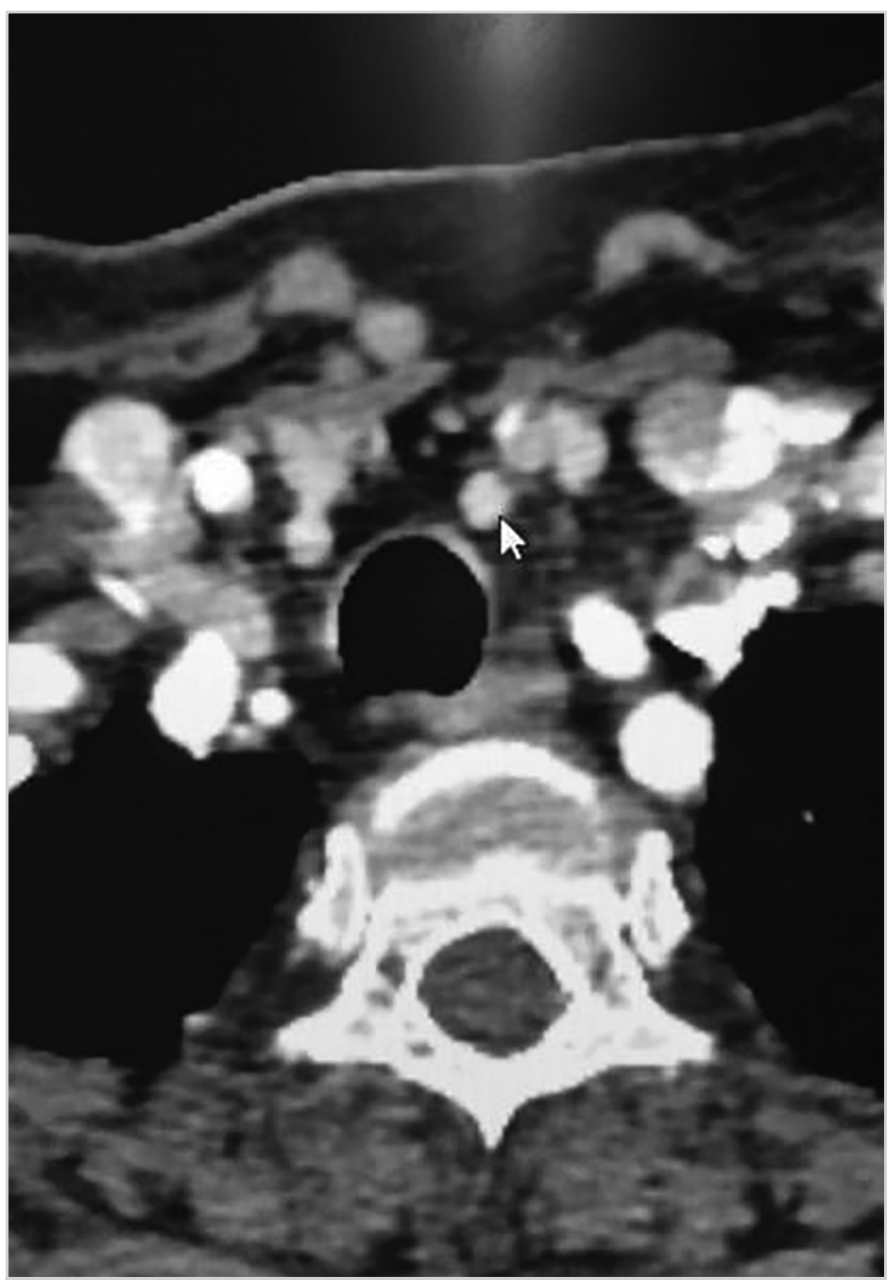

Figure 3. A four-dimensional parathyroid CT (4D-CT) revealed a $15 \times 7 \mathrm{~mm}$ PTA which was similar to the cranial cephalic artery in the left thyroid loach inferior

A universal algorithm for parathyroid imaging is still not available. Based on imaging expertise and surgeon preference, the use of USG and MIBI as the first-line modalities is common. However, USG is insufficient in revision surgeries and in iden- tifying ectopic parathyroid gland, small lesions, multigland disease. Thyroid nodules and lymph nodes may imitate adenomas in USG. USG could not localize ectopic parathyroid in any of our patients. In case 2, we employed MIBI with a hand-held gamma probe to improve surgical success. Some surgeons combine gamma probe and intraoperative PTH assay but the efficacy of this is still unclear (5).

Single photon emission computed tomography (SPECT) is increasingly used in parathyroid imaging to complement MIBI. Three dimensional scans better detect difficult ectopic PTA, small lesions and depth of lesion within thyroid gland (6). Combining SPECT with CT (SPECT-CT) provides additional anatomic information (4). In case 5, SPECT-CT failed to localize the lesion. In this case we used $4 \mathrm{D}-\mathrm{CT}$, with success. In the past two years, we have used 4D-CT scanning as the final imaging method in seven patients with negative USG and negative MIBI scans. This technique is particularly advantageous in detecting multigland disease, persistent or recurrent disease. Although no imaging modality is superior to the other in the determination of parathyroid lesions, the popularity of 4D-CT scanning is increasing (4). There is no clear source of PTA in a small but significant minority of patients, including multigland disease, persistent or recurrent disease, despite multiple imaging. $4 \mathrm{D}-\mathrm{CT}$ scanning has advantages compared to other modalities in patients including multigland disease, persistent or recurrent disease (4). In our center, 4D-CT scanning has been used for two years. Tomography-guided preoperative localization was confirmed during surgery. The sensitivity of this technique was confirmed by the success of the surgery.

BNE has been the gold standard operation for sporadic PHPT (7). Negative imaging studies before the surgery or suspicion of multigland disease are certain indications for BNE. Using intraoperative PTH may prevent the necessity of BNE in many patients. Surgeons routinely performing BNE with intraoperative parathormone monitoring have shown that anatomically enlarged glands may remain in situ despite a possible imme- 
diate drop in intraoperative PTH levels following the excision of the imaged-targeted gland (8). Surgeons must determine the best course of action in each patient, using their judgment, experience, and skills, until there is better understanding of overproduction and parathyroid enlargement. Gasparri (8) stated that at least a decade was needed to compare BNE with focused surgeries using intraoperative PTH. This is because some of the glands may be in sleep during the surgery and be responsible for late recurrence. Norman et al. (9) abandoned unilateral parathyroidectomy after they experienced a 10 -year recurrence rate of $4-6 \%$ and 1-year failure rate of $3-5 \%$ with this technique. Besides, intraoperative PTH assays are extremely expensive. This is a major problem for developing countries such as Turkey. Horányi et al. (10) reported that minimally invasive procedures might not detect other overactive gland(s) suppressed in the presence of dominant gland(s). Therefore, they recommended other intraoperative methods such as histopathologic evaluation of frozen sections. The confirmation of the diagnosis was made using frozen-section examinations instead of intraoperative PTH in our patients. With improvement in preoperative imaging, focused unilateral neck exploration (FUNE) has become the preferred approach. For ectopic parathyroid pathology, the surgical procedure varies based on imaging results and intraoperative findings. A systematic BNE is necessary when imaging does not detect the abnormalities, or when abnormality imaging is not present during surgery (missing parathyroid glands).

\section{Conclusion}

Our case series shows that in addition to preoperative imaging results, the preparedness and the experience of the surgeon in converting FUNE to BNE with or without mediastinal exploration, lobectomy, or total thyroidectomy is crucial for the successful management of ectopic parathyroid pathologies.

Informed Consent: Written informed consent was obtained from the patients.

Peer-review: Externally peer-reviewed.
Author Contributions: Concept - A.B., B.D.; Design - A.B.; Supervision - A.B., B.D.; Resource - B.D, J.M.; Data Collection and/or Processing - J.M.; Analysis and/or Interpretation - A.B., B.D.; Literature Search - B.D., J.M.; Writing - A.B., B.D.; Critical Reviews - A.B., B.D.

Conflict of Interest: The authors have no conflicts of interest to declare.

Financial Disclosure: The authors declared that this study has received no financial support.

\section{References}

1. Binnetoglu A, Gundogdu Y, Baglam T, Sari M. A surgical challenge for primary hyperparathyroidism: Intravagal parathyroid adenoma. Arch Otolaryngol Rhinol 2018; 4: 57-60.

2. Lumachi F, Zucchetta P, Varotto S, Polistina F, Favia G, D'Amico D. Noninvasive localization procedures in ectopic hyperfunctioning parathyroid tumors. Endocr Relat Cancer 1999; 6: 123-5. [Crossref]

3. Noussios G, Anagnostis P, Natsis K. Ectopic parathyroid glands and their anatomical, clinical and surgical implications. Exp Clin Endocrinol Diabetes 2012; 120: 604-10. [Crossref]

4. Kuzminski SJ, Sosa JA, Hoang JK. Update in parathyroid imaging. Magn Reson Imaging Clin N Am 2018; 26: 151-66. [Crossref]

5. Flynn MB, Bumpous JM, Schill K, McMasters KM. Minimally invasive radioguided parathyroidectomy. J Am Coll Surg 2000; 191: 24-31. [Crossref]

6. Spanu A, Schillaci O, Piras B, Madeddu G. SPECT/CT in hyperparathyroidism. Clin Transl Imaging 2014; 2: 537-55. [Crossref]

7. Kelly HR, Hamberg LM, Hunter GJ. 4D-CT for preoperative localization of abnormal parathyroid glands in patients with hyperparathyroidism: accuracy and ability to stratify patients by unilateral versus bilateral disease in surgery-naive and re-exploration patients. AJNR Am J Neuroradiol 2014; 35: 176-81. [Crossref]

8. Gasparri G. Updates in primary hyperparathyroidism. Updates Surgery 2017, 69: 217-23. [Crossref]

9. Norman J, Lopez J, Politz D. Abandoning unilateral parathyroidectomy: Why we reversed our position after 15,000 parathyroid operations. J Am Coll Surg 2012; 214: 260-69. [Crossref]

10. Horányi J, Duffek L, Szlávik R, Darvas K, Lakatos P, Tóth M, et al. Parathyroid surgical failures with misleading falls of intraoperative parathyroid hormone levels. J Endocrinol Invest 2003; 26: 1095-9. [Crossref] 